\title{
Development of an Autonomous Mobile Health Monitoring System for Medical Workers with High Volume Cases
}

\author{
Ajiroghene $\mathrm{O}^{1}$, Obiei-uyoyou $\mathrm{O}^{1}$, Chuks $\mathrm{M}^{1}$, Ogaga $\mathrm{A}^{2}$, Chukwumenogor $\mathrm{O}^{2}$, Elvis $\mathrm{R}^{2}$, Udoka ED³, Bright $\mathrm{AE}^{3}$, Ofualagba $\mathrm{G}^{1}$ and Ejofodomi \\ OA $^{1 *}$
}

${ }^{1}$ Department of Electrical and Electronics Engineering, Federal University of Petroleum Resources (FUPRE), Nigeria

${ }^{2}$ Department of Mechanical Engineering, Federal University of Petroleum Resources (FUPRE), Nigeria

${ }^{3}$ Department of Marine Engineering, Federal University of Petroleum Resources (FUPRE), Nigeria

\begin{abstract}
Mobile health care monitoring system have the capacity to provide critical assistance in areas of medical care, especially in situations where there are limited number of health workers needed to attend to patients. This project describes the development of a mobile health care monitoring system capable of monitoring the heart rate of patients and recording this information electronically. The system will provide much needed relief for medical workers with high volume cases. The system was constructed using a mobile robot capable of traversing rugged terrain, a motor shield, a Global Positioning System (GPS) shield for autonomous navigation, an ultrasound sensor for obstacle avoidance, and a heart rate sensor for measuring patient heart rate. The mobile system was able to successfully avoid obstacles and navigate to the predetermined locations of three hospital beds and obtain the heart rate of three patients. Future improvements to the system include the addition of wireless capability so patient data can be transmitted wirelessly to physician's PC, incorporation of speech capability to enhance interaction between robot and patient and addition of more sensors to measure other vital signs such as blood glucose.
\end{abstract}

Keywords: Global positioning system (Gps); Mobile health; Medical workers

\section{Introduction}

Mobile health care monitoring system have the capacity to provide critical assistance in areas of medical care, especially in situations where there are limited number of health workers needed to attend to patients, for instance in a united nation refugee camp where there is shortage of medical staff and resources are scarce. It is important to always monitor the vital signs of patients as this gives a very good idea of their medical condition.

Currently mobile health monitoring systems typically consists of wearable biomedical sensors that are attached to a person's physical body. Relevant biological signals are acquired by these sensors and the data is transferred wirelessly to a PC. Shahriyar et al. [1] developed an Intelligent Mobile Health Monitoring System (IMHMS) that uses the Wearable Wireless Body/Personal Area Network for collecting data from patients, mining the data, intelligently predicts patient's health status and provides feedback to patients through their mobile devices. The acquired biological data is stored in the patient's mobile device and is then sent to an intelligent automated medical server for analysis. The automated server will then provide feedback to the patient based on the data it receives. This system encourages patients to participate in the health care process using their mobile devices and patients are able to access their health information from anywhere and at any time. IMHMS is intended to be used by patients who have been discharged from the hospital but still need to constantly monitor vital biological signals. It is not intended to be used in the hospital setting.

Ziyu Lv et al. [2] have developed a mobile health monitoring system called iCare, which is to be used by older people anytime anywhere. The iCare uses body sensors and devices to collect physiological signals from the elderly and transmit them to a smart phone that will process physiological data locally and send an alarm automatically to the emergency centre and pre-assigned people when the data exceeds the threshold of the fixed device. The emergency centre will call an ambulance to the current location of the elderly. The location information can be gained from the alarm message sent to the emergency centre. Sensors and devices can be tailored depending on the old people's health condition. The physiological data will be not only analyzed locally, but also sent to the server in bulk to construct the personal health information system. With viewing the current and history condition of the old people in the personal health information system, doctors remotely set the thresholds and give advices which can guide the old people to adjust themselves to health mode [2]. The iCare also offers auxiliary functions such as the life assistant, which provides services like reminders and location tracking. The iCare system is to be used to provide remote medical monitoring services to older patients in their homes, and not for patients in the hospital setting.

Many other mobile health care monitoring systems have been developed [3-11]. However, the major design concept remains the same: patient is fitted with wearable biomedical sensors outside the hospital setting and vital signs are measured and transferred wirelessly either to a mobile device or to a server. Acquired data is either reviewed by medical professionals for remote medical monitoring, or by an intelligent medical server as is the case for IMHMS. All these health care monitoring systems have been developed to be used outside the hospital setting.

*Corresponding author: Ejofodomi OA, Department of Electrical and Electronics Engineering, Federal University of Petroleum Resources (FUPRE), Nigeria, Tel +234 703919 0064; E-mail: tegae@yahoo.com

Received August 31, 2015; Accepted October 13, 2015; Published October 15 2015

Citation: Ajiroghene O, Obiei-uyoyou O, Chuks M, Ogaga A, Chukwumenogor O et al. (2015) Development of an Autonomous Mobile Health Monitoring System for Medical Workers with High Volume Cases. Adv Robot Autom S2: 005. doi: 10.4172/2168-9695.S2-005

Copyright: (c) 2015 Ajiroghene O, et al. This is an open-access article distributed under the terms of the Creative Commons Attribution License, which permits unrestricted use, distribution, and reproduction in any medium, provided the original author and source are credited. 
The mobile health care monitoring system presented in this paper takes a different approach. It has been designed specifically to be used in the hospital setting to provide assistance to medical workers dealing with high volume cases. The biomedical sensors used in this system are not are mounted on a mobile robot, which autonomously moves from patient to patient. This system affords the opportunity to monitor multiple patients and will provide much needed relief for medical workers, especially in developing countries.

Blood pressure is a very vital health parameter that is directly related to the soundness of the human cardiovascular system. High blood pressure is often an important risk factor for many diseases [12]. The mobile health care monitoring system presented in this paper monitors the heart rate of patients and records this information electronically. It uses a technique of measuring heart rate through the fingertip by means of an Arduino development board in conjunction with a mobile robot. When the heart beats, it pumps blood throughout the body, causing the blood volume inside the arteries of the fingers to change too. This fluctuation of blood can be detected through a pressure sensing mechanism on which the fingertip is placed. The signal can be amplified further for a microcontroller to count the rate of fluctuation, which is actually the heart beat rate. The arduino heart rate meter computes the heart beat rate by processing the analog pulse signal output from the sensor. Heart rate values are stored electronically in a memory card that can be accessed by the physician once the mobile system has completed its rounds. The design and construction of the automated mobile health monitoring system is presented in the materials and methods section. Analysis of the system's design, its advantages and limitations are discussed in the discussion and conclusion section. Future improvements to the system are also mentioned briefly.

\section{Materials and Methods}

\section{Materials}

Robot chassis: The robot chassis used in this research project was a four wheel drive chassis from Dagu Electronics (Figure 1). It is designed to drive on rough terrain, making it a great platform for robots that need to perform tasks in outdoor environments. The size of the robot is 11 by 12 by 5 inches. The movement of the robot was provided by four DC motors with brass brushes and 75:1 steel gearboxes that drive $120 \mathrm{~mm}$ diameter spiked tires. These motors are designed to run between $2 \mathrm{~V}$ and $7.2 \mathrm{~V}$. The chassis can reach a top speed of approximately 3 kilometers per hour $(\mathrm{km} / \mathrm{hr})$ when powered at $7.2 \mathrm{~V}$. The stall torque of each motor is roughly around $11 \mathrm{~kg}-\mathrm{cm}$. The chassis has a spring suspension system which keeps each wheel in contact with the ground for maximum traction. The chassis is made from a $2 \mathrm{~mm}$ thick corrosion-resistant anodized aluminum plate, and the nuts and bolts are made from stainless steel [13].

Arduino Uno R3: The arduino board (Figure 2) serves as the

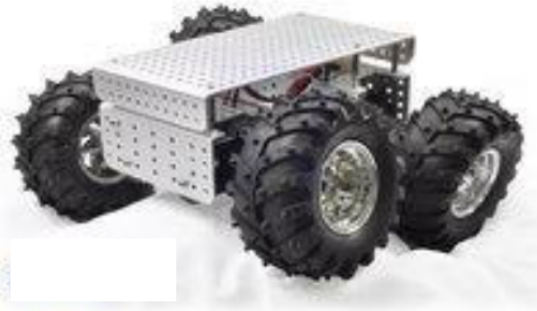

Figure 1: Chassis for Autonomous Mobile Health Monitoring System.

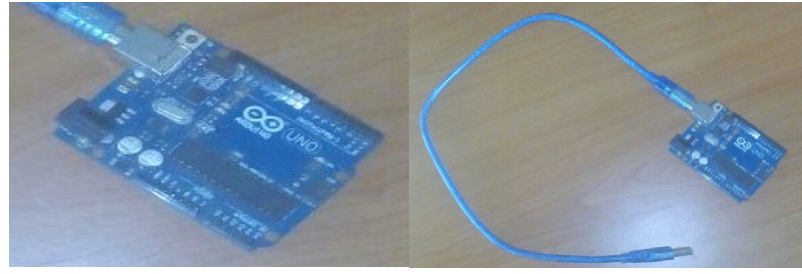

Figure 2: Arduino board with USB cable attached.

(a) MegaMoto Shield

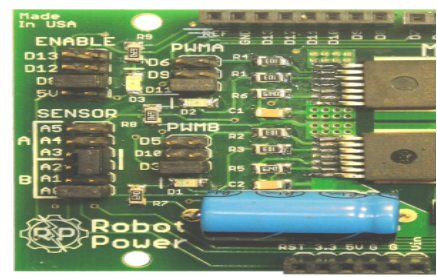

(b) Moto shield mounted on robot chassis.

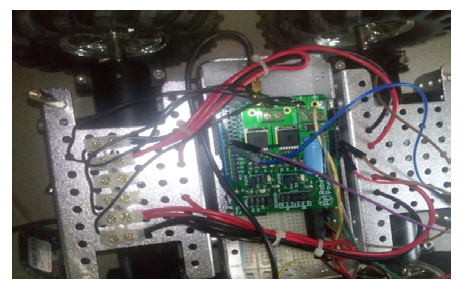

Figure 3: Robot Power Mega Moto Shield.

brain for this mobile health monitoring system. It processes all input and output system of the robotic system as well as all the integrated circuits connected for specific operation of the robotic system such as GPS, heart rate sensor and ultrasonic sensor. The USB cable was used to burn the code developed in a computer into the microcontroller on the arduino board.

Robot power mega moto shield: The motor shield was used to control the geared motor system, which in turn controls the wheels of the robot (Figure 3 ). The motor shield was powered by an external battery because the Arduino microcontroller supplies a maximum current of $40 \mathrm{~mA}$ which is insufficient to drive the motor of the mobile robot. The MegaMoto circuit is two independent half-bridge circuits mounted on a single PCB. The two motor outputs may work together to form a full $\mathrm{H}$-bridge for bi-directional drive of a single motor or other load or each half-bridge may be used independently. When connected in half-bridge mode the MegaMoto may be used for driving uni-directional loads such as lamps, heaters, solenoids or DC motors in one direction. In this research project, a single mega moto shield was connected in half-bridge mode to drive to two pairs of DC motor. In this configuration, the motor is not enabled to do a reverse movement. To enable a reverse movement, two mega moto shields will be needed [14].

GPS shield: The Arduino GPS shield is a GPS module breakout board designed for Global Positioning System receiver with SD interface (Figure 4). It can be used to read position data of the fire detection system and can also store the data in the SD card. The $5 \mathrm{~V} / 3.3 \mathrm{~V}$ compatible operation voltage level makes it compatible with Arduino boards. The GPS shield was used for determining the GPS 


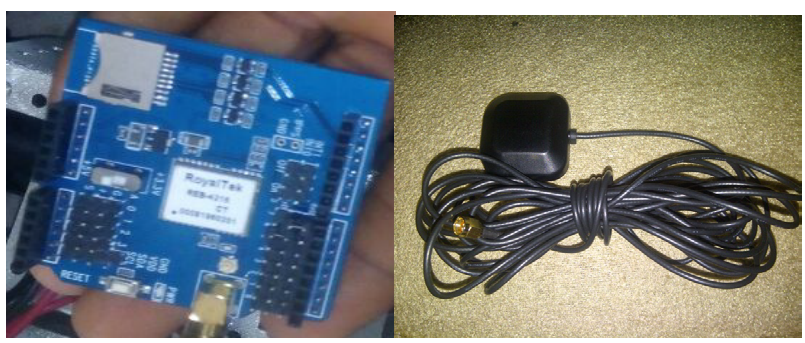

Figure 4: Global Positioning System (GPS) shield for Arduino.

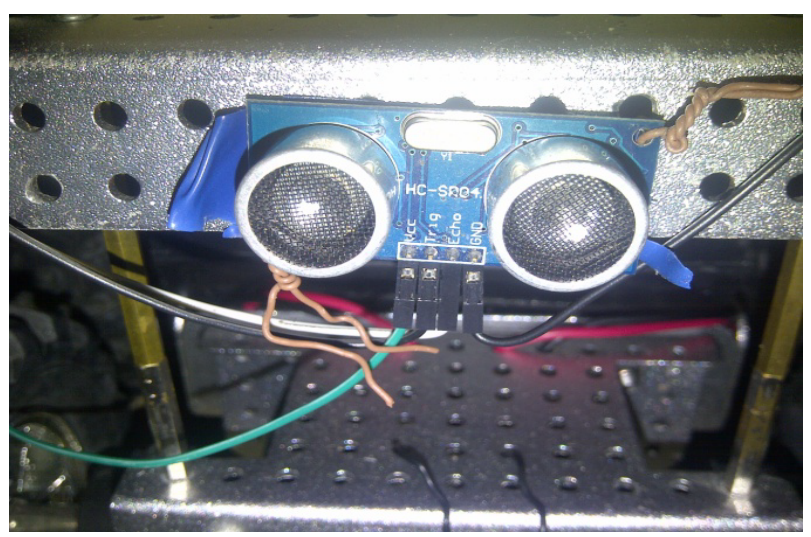

Figure 5: Ultasonic sensor mounted in front of robot for autonomous navigation

location of the position where any operation will be carried out by the robot. A GPS antenna magnetic mount SMA was also used for sending and transmitting information containing fire location. The GPS shield is suitable for automotive navigation, personal positioning, fleet management and marine navigation [15].

Ultrasonic sensor: The ultrasonic sensor was used to detect the obstacles at a certain distance from the mobile robot (Figure 5). An ultrasonic pulse is sent out. The time taken to receive an echo signal by the sensor is used to estimate the distance between the robot and an obstacle. If the obstacle is close to the robot, the program instructs the robot to take an alternate path.

Hear rate sensor: The heart rate sensor is an integrated circuit device that detects heat rate in a human.

Buzzer: A piezo buzzer was used to give out a sound corresponding to a particular frequency. This served as the alarm signal if the measured heart rate was above a certain threshold.

\section{Methods}

All hardware device and circuitry used were connected to the Arduino Uno R3 microcontroller board. The different component parts connected were made as shown in the block diagram below (Figure 6).

The megamoto shield was stacked on the arduino uno and the gps shield was mounted on the megamoto shield (Figure 7). The arduino uno was powered by a 9 volts battery supply system and served as the power supply for the heart rate monitoring circuit, buzzer circuit and GPS shield. The megamoto shield was powered by a rechargeable 12 volts lead acid battery and was used to control for the geared motors which were responsible for movement of the mobile robot. Ultrasonic sensor was connected to the arduino and mounted in front of the robot (Figure 5) to aid obstacle avoidance. The heart rate sensor and the piezo buzzer were also connected to the arduino and mounted at the front of the robot. The schematic of how the mobile health monitoring system functions is shown in Figure 8. The robot is programmed to navigate a pre-defined route. For testing purposes it was assumed that the system would be recording the heart rate of only three patients in a hospital setting. The GPS locations of the three hospital beds are stored within the system's program. The mobile system begins its rounds from the physician's office. The ultrasonic sensor turns $\mathrm{ON}$ as the robot moves. The ultrasonic sensor detects any object at a distance and sends signals to the microcontroller which sends signals to the motor shield to turn the robot in a different direction.

The robot navigates until it arrives at the GPS location of the first hospital bed. It then gives a buzzing sound to prompt the patient to place his or her finger tip on the sensor. After sensor measures the heart rate of the patient, the value is stored in the SD card in the GPS shield. If the patient fails to place his or her finger tip on the sensor, a specific value is recorded, indicating the absence of a reading. The entire process is repeated for the second and third hospital bed. Once the heart rates of the three patients have been recorded, the robot navigates back to the physician's office. The physician can then extract the SD card from the system to view the heart rate log file.

\section{Results and Discussion}

The mobile health monitoring system was assembled and tested at the Student's Center in the Federal University of Petroleum Resources.

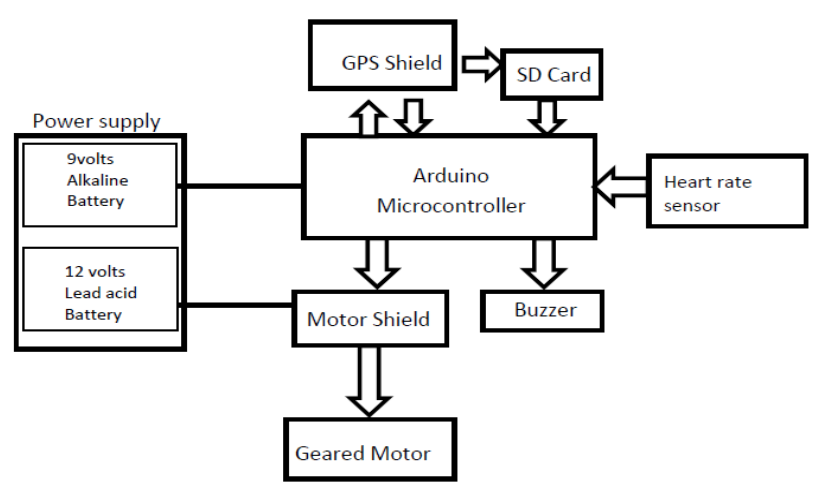

Figure 6: Block Diagram of Mobile Health Monitoring System.
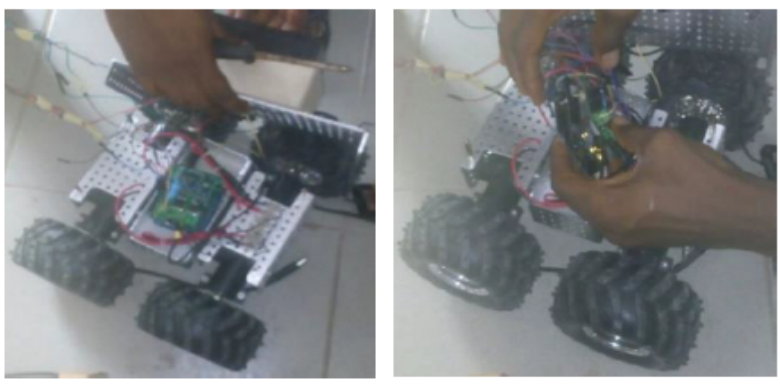

Figure 7: Assembling the autonomous mobile health monitoring system. 


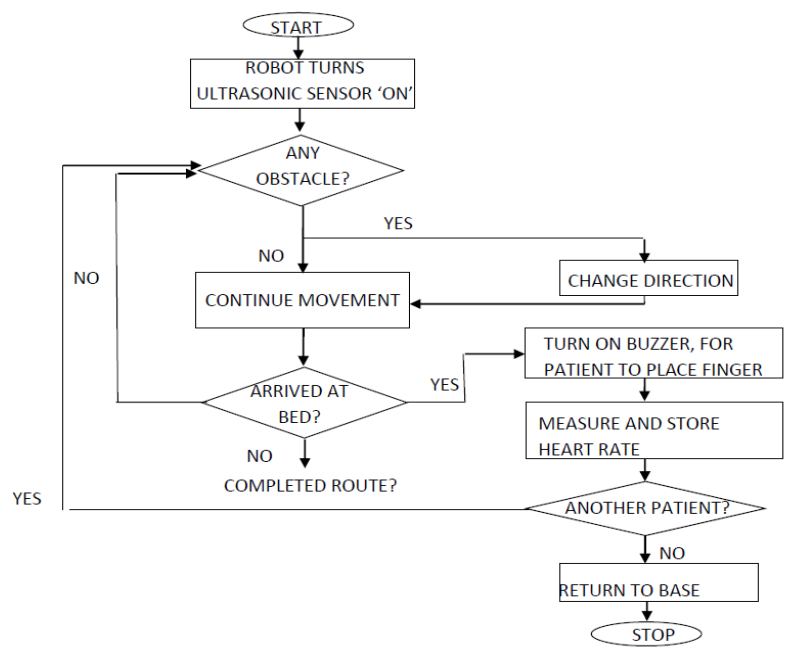

Figure 8: Flow Chart of Mobile Health Monitoring System.

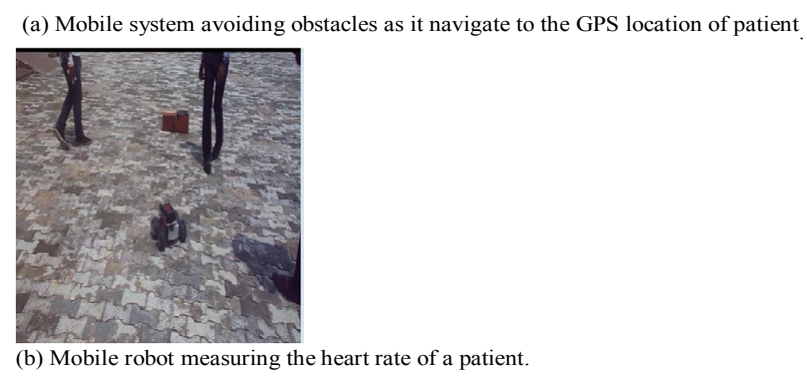

(b) Mobile robot measuring the heart rate of a patient.

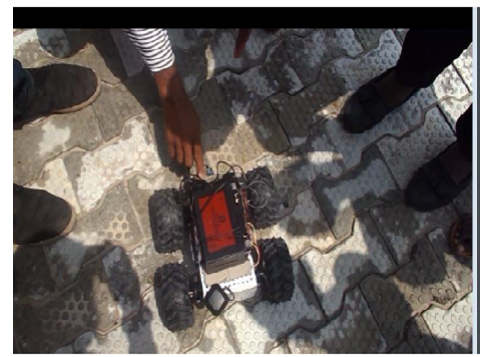

(c) Viewing Patient data stored in SD card of the system

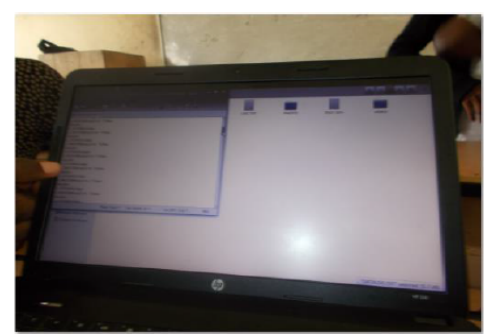

Figure 9: Autonomous mobile health monitoring system.

The ability to avoid obstacles was successfully demonstrated (Figure 9a) by placing a set of brick blocks in the path of the robot. The robot turned left away from its straight path, moved along that direction for a second before turning right towards its normal path, continuing its movement before avoiding the second obstacle in a similar pattern. It then moved to the place where the three patients were standing and gave a buzz sound for the first patient to place his fingertip, measured and recorded the heart rate, moved to the second patient, measured and recorded the heart rate and then did the same for the third patient (Figure 9b). After this the robot returned to its starting point, The memory card was removed and inserted into a computer where the results of the heart rate were indicated as high, normal and low with corresponding values of their heart rate in volts (Figure 9c).

To our knowledge, this is the first mobile health monitoring system that is being developed to be used within a hospital setting. As mentioned in the previous section, all mobile health monitoring systems currently being developed are being used outside of the hospital setting for remote health care monitoring by the patient. This system, on the other hand, has been designed to be used by the medical professionals in hospitals. To our knowledge it is also the first health monitoring system that has locomotive capabilities. The results presented in this paper are from the initial design and testing of this new health monitoring system. It is expected that subsequent versions of this system will demonstrate a more robust design and higher complexity.

The mobile robot used as the base of the health monitoring system is only about 5 inches high. This is not ideal for obtaining blood pressure measurements from patients lying in hospital beds. This limitation can be addressed in one of two ways. The first is to use a larger mobile robot with about the same height as the hospital bed. The second alternative is to install long rods on the existing robot on which the biomedical sensors can be attached. The height of the rods can be adjusted to the position that is most comfortable for the patient.

The primary focus for the initial testing of the system was to ensure that the robot could autonomously go to the patient's bedside, obtain the patient's blood pressure, save that data in its onboard memory, and return back to the physician with the acquired data. This was successfully demonstrated by the system. However, there are several improvements that will be made to the mobile health monitoring system. Wireless capability will be incorporated into the system so that the patient data are transmitted wirelessly to the physician's PC without the physician needing to remove the SD card to acquire the data. This could improve the response time of the physician in cases where urgent attention is needed. The system will also be given speech capability to enhance interaction between robot and patient so as to ensure accurate readings are obtained. The ultimate goal is to have a humanoid robot capable of assisting medical workers in the hospital setting and so robot-patient interaction should be identical to doctorpatient interaction.

There will be inclusion of more sensors and other devices to obtained more detailed medical information about the patient. Presently, the mobile health monitoring system is only capable of measuring and detecting patient heart rate, but it could be upgraded to measure other vital signs such as blood glucose content for diabetic patients. A video camera will also be integrated into the system to provide visual inspection of the patient. Future tests on the automated health monitoring system will be conducted inside of a hospital, as it is intended to be used in hospital settings.

\section{Conclusion}

Mobile health care monitoring systems developed to date are meant to be used outside the hospital settings. Patients are fitted with wearable biomedical sensors, and vital signs are measured and transferred wirelessly either to a mobile device or to a server for remote medical monitoring. The mobile health care monitoring system 
Citation: Ajiroghene O, Obiei-uyoyou O, Chuks M, Ogaga A, Chukwumenogor O, et al. (2015) Development of an Autonomous Mobile Health Monitoring System for Medical Workers with High Volume Cases. Adv Robot Autom S2: 005. doi: 10.4172/2168-9695.S2-005

Page 5 of 5

presented in this paper has been designed specifically to be used in the hospital setting to provide assistance to physicians dealing with high volume cases. The biomedical sensors used in this system are not are mounted on a mobile robot, which autonomously moves from patient to patient. This system affords the opportunity to monitor multiple patients and will provide much needed relief for medical workers, especially in developing countries. To our knowledge, this is the first health monitoring system being developed to be used in the hospital setting. It is also the first automated health monitoring system with locomotive capabilities. The system was able to successfully measure and store heart rates from three patients in three different locations. The ultimate goal is to develop a humanoid robot capable of assisting physicians with high volume cases in developing countries.

\section{References}

1. Shahriyar R, Bari F, Kundu G, Ahamed SI, Akbar M (2009) Intelligent Mobile Health Monitoring System (IMHMS). International Journal of Control and Automation 2: 13-28.

2. Lv Z, Xia F, Wu G, Yao L, Chen Z (2010) I Care: A Mobile Health Monitoring System for the Elderly. Physical and Social Computing (CPSCom) 1: 699-705.

3. Bourouis A, Feham M, Bouchachia A (2011) Ubiquitous Mobile health Monitoring System for Elderly (UMHSE). International Journal of Computer Science and Information Technology (IJCSIT) 3: 1-3

4. Jovanov E, Milenkovic A, Otto C, De Groen PC (2005) A wireless body area network of intelligent motion sensors for computer assisted physical rehabilitation. Journal of NeuroEngineering and Rehabilitation 2: 1-6.

5. Konstantas D, Halteren AV, Bults R, Wac K, Jones V, et al. (2004) Mobile health:
Ambulant Patient Monitoring Over Public Wireless Networks. Mediterranean Conference on Medical and Biological Engineering.

6. Choi JM, Choi BH, Seo JW, Sohn RH, Ryu MS, et al. (2004) System for Ubiquitous Health Monitoring in the Bedroom via a Bluetooth Network and Wireless LAN. Engineering in Medicine and Biology Society 2: 3362-3365.

7. Farella E, Pieracci A , Brunelli D, Benini L, Ricco B, et al. (2005) Design and implementation of WiMoCA node for a body area wireless sensor network. Proceedings of the 2005 Systems Communications 2: 342-347.

8. Morón MJ, Luque JR, Botella AA, Cuberos EJ, Casilari E (2007) A Smart Phone-based Personal Area Network for Remote Monitoring of Biosignals. $4^{\text {th }}$ International Workshop on Wearable and Implantable Body Sensor Networks 13: $116-121$.

9. Dai S, Zhang Y (2006) Wireless Physiological Multi-parameter Monitoring System Based on Mobile Communication Networks. 19th IEEE Symposium on Computer-Based Medical Systems Based on Mobile Communication Networks, Washington.

10. Lee JW, Jung JY (2007) ZigBee Device Design and Implementation fo Context-Aware UHealthcare System. The IEEE 2nd International Conference on Systems and Networks Communications, Cap Esterel, French.

11. Yang G (2006) Body Sensor Networks. (1stedn), Springer.

12. Lloyd-Jones D, Adams R, Carnethon M (2009) Heart disease and stroke statistics-update: a report from the American heart association statistics committee and stroke statistic subcommittee. Circulation 119: 40-41.

13. www.dagurobot.com

14. www.robotpower.com

15. www.iteadstudio.com
This article was originally published in a special issue, entitled: "Robotics in Surgery", Edited by Buchs Nicolas, University Hospitals of Geneva, Switzerland 